PUPT-1881

ITEP-TH-38/99

CALT-68-2244

CITUSC/005

\title{
Solitons, Superpotentials and Calibrations
}

\author{
Sergei Gukovil \\ Joseph Henry Laboratories, Princeton University \\ Princeton, New Jersey 08544 \\ and \\ California Institute of Technology, Pasadena, California 91125 \\ CIT-USC Center For Theoretical Physics
}

\begin{abstract}
In this paper we study several issues related to the generation of superpotential induced by background Ramond-Ramond fluxes in compactification of Type IIA string theory on Calabi-Yau four-folds. Identifying BPS solitons with D-branes wrapped over calibrated submanifolds in a Calabi-Yau space, we propose a general formula for the superpotential and justify it comparing the supersymmetry conditions in $D=2$ and $D=10$ supergravity theories. We also suggest a geometric interpretation to the supersymmetric index in the two-dimensional effective theory in terms of topological invariants of the Calabi-Yau four-fold, and estimate the asymptotic growth of these invariants from BTZ black hole entropy. Finally, we explicitly construct new supersymmetric vacua for Type IIA string theory compactification on a Calabi-Yau four-fold with Ramond-Ramond fluxes.
\end{abstract}

November 1999

1 On leave from the Institute of Theoretical and Experimental Physics and the L. D. Landau Institute for Theoretical Physics 


\section{Introduction and Summary}

The theory of calibrations was developed by Harvey and Lawson [1] for the purpose of obtaining geometries with the property that all varieties in the geometry are volume minimizing. Due to this fundamental property, calibrations have proven to be a very useful tool for investigating supersymmetric configurations of D-branes in string theory. A partial list of references includes [2-9]. Although a significant progress has been made in the study of the local differential geometry of the moduli space of calibrated submanifolds [10]11], its global structure remains elusive. Motivated by [12,13], we hope that physical applications of calibrated geometry can help to address this problem.

In this paper we study compactification of Type IIA string theory on a Calabi-Yau four-fold with background Ramond-Ramond fluxes. In particular, we are interested in the vacuum and soliton structure of the resulting $\mathcal{N}=(2,2)$ theory in two dimensions. We show that supersymmetric vacua can be described by the effective superpotential generated by Ramond-Ramond fields, while the BPS solitons connecting these vacua correspond to D-branes wrapped over supersymmetric cycles in the Calabi-Yau space $X$. Using the relation between supersymmetric cycles and calibrations we derive the general formula for the superpotential in the effective field theory:

$$
W=\sum \int_{X}(\text { calibrations }) \wedge(\text { Ramond }- \text { Ramond fields })
$$

There are two basic types of calibrations on a Calabi-Yau four-fold $X$ given by the covariantly constant forms $\operatorname{Re}(\Omega)$ and $\frac{1}{p !} \mathcal{K}^{p}$, where $\Omega$ denotes the nowhere-vanishing holomorphic $(4,0)$-form and $\mathcal{K}$ is the Kähler form on $X$. The first type of calibrations corresponds to special Lagrangian submanifolds in $X$ and leads to a chiral superpotential, while the latter is associated with holomorphic cycles of complex dimension $p$ and generates a twisted chiral superpotential.

Given the induced superpotential $W$ one can define a supersymmetric index $\mathcal{I}_{[S]}(X)$ that counts the "number" of supersymmetric cycles with the homology class $[S] \in H_{*}(X)$. Namely, $\mathcal{I}_{[S]}(X)$ is equal to the Euler number of the moduli space of calibrated submanifolds $S$ with flat line bundles:

$$
\mathcal{I}_{[S]}(X)=\chi\left(\mathcal{M}_{[S]}(X)\right)
$$

We argue that $\mathcal{I}_{[S]}(X)$ is a topological invariant of the Calabi-Yau four-fold $X$. The dimension of $X$ is very important here, so we do not expect $\mathcal{I}_{[S]}(X)$ to generalize directly 
to higher (or lower) dimensional Calabi-Yau manifolds (however, see [14] and [15] where analogous invariants of Calabi-Yau three-folds have been discussed). In some special cases, $\mathcal{I}_{[S]}(X)$ can be shown to agree with classical topological invariants of $X$. For example, when $[S]$ is the class of a special Lagrangian torus, from the conjecture of Strominger, Yau and Zaslow [12] it follows that $\mathcal{I}_{[S]}(X)=\chi(X)$.

The paper is organized as follows. In the next section we describe the relevant aspects of $\mathcal{N}=(2,2)$ theories constructed from Calabi-Yau four-folds and derive the effective superpotential generated by Ramond-Ramond fluxes. In section 3 we identify BPS solitons in the effective theory with D-branes wrapped over calibrated submanifolds in Calabi-Yau spaces. We also discuss possible generalizations and conjecture the form of the superpotential generated by background fields in compactification on manifolds with exceptional holonomy groups, $G_{2}$ and $\operatorname{Spin}(7)$. Superpotentials induced by membrane instantons in $G_{2}$-manifolds have been discussed in [16] and [17]. Section 4 is devoted to the geometrical interpretation of the supersymmetric index $\mathcal{I}_{[S]}(X)$. In the case when $S$ is a holomorphic curve, we estimate the asymptotic growth of $\mathcal{I}_{[S]}(X)$ from the counting of BTZ black hole microstates. In section 5 we explicitly construct new supersymmetric vacua for Type IIA string theory compactification on Calabi-Yau four-folds with Ramond-Ramond fluxes. A work along these lines has been presented recently in [18]. Finally, in the appendix we justify the formula for the induced superpotential comparing the supersymmetry conditions in $D=2$ and $D=10$ supergravity theories.

Throughout the paper we assume that $X$ is a smooth compact Calabi-Yau four-fold, so that all the vacua in the effective two-dimensional theory are connected by solitons. Most of the results presented here can be generalized to a non-compact $X$, though not all the vacua are connected by solitons in such models [19].

\section{Superpotentials from Calabi-Yau Four-folds}

Compactification of Type IIA string theory on a Calabi-Yau four-fold $X$ leads to $\mathcal{N}=$ $(2,2)$ theory in two dimensions. The low-energy spectrum of this theory includes $h^{3,1}$ chiral superfields $\Phi_{i}$ and $h^{1,1}$ twisted chiral superfields $\Sigma_{j}$. Here we use the standard notation $h^{p, q}$ for the dimension of the Hodge group $H_{\bar{\partial}}^{p, q}(X)$. We denote by $\phi_{i}$ and $\sigma_{j}$ the scalar components of the superfields $\Phi_{i}$ and $\Sigma_{j}$ which correspond, respectively, to deformations of the complex and the Kähler structure of $X$. Note, that the mirror symmetry maps Type 
IIA string theory on a four-fold $X$ also to Type IIA string theory on the mirror variety $\widetilde{X}$, such that:

$$
h^{p, q}(X)=h^{4-p, q}(\widetilde{X})
$$

and conformal field theories associated with $X$ and $\widetilde{X}$ are equivalent. In the twodimensional effective theory this operation corresponds to the twist that exchanges the multiplets $\Phi_{i}$ and $\Sigma_{j}$.

Let us assume for a moment that there are no background fluxes. Then the twodimensional effective theory is described by $\mathcal{N}=(2,2)$ dilaton supergravity interacting with a non-linear sigma-model. The complete superspace formulation of this theory will be presented elsewhere [20]. The target space of this sigma-model is parametrized by the chiral fields $\left\{\phi_{i}\right\}$ and the twisted chiral fields $\left\{\sigma_{j}\right\}$, so that the target space metric is Kähler and torsionless. From the Kaluza-Klein reduction of Type IIA theory on $X$ one can easily find that in the large volume limit the target space metric is equal to the metric on the moduli space of $X, \mathcal{M}_{c}(X) \times \mathcal{M}_{\mathcal{K}}(X)$. For example, the effective Kähler potential for the chiral superfields:

$$
K_{c}\left(\Phi_{i}, \bar{\Phi}_{\bar{i}}\right)=-\log \left(\int_{X} \Omega \wedge \bar{\Omega}\right)
$$

where $\Omega$ denotes the covariantly constant $(4,0)$-form, is equal to the Kähler potential of the Weil-Petersson metric on $\mathcal{M}_{c}(X)$. Therefore, at least classically, we may identify the space of vacua with the moduli space of the Calabi-Yau manifold $X$. However, as we explain below, most of these vacua are lifted once we turn on Ramond-Ramond fluxes.

One of the most important features of low-dimensional string theory compactifications is the global anomaly [21,22] given by the Euler number, $\chi(X)$, of the Calabi-Yau fourfold $X$. In general, to cancel the anomaly one has to introduce a background flux for the four-form field $G$ and/or $N$ fundamental strings filling two-dimensional space-time, such that the following condition is satisfied:

$$
N=\frac{\chi}{24}-\frac{1}{2(2 \pi)^{2}} \int_{X} G \wedge G
$$

In particular, $G$ must be non-zero when $\chi(X) / 24$ is not integer. Besides $G$-fluxes one can also turn on background values of the other Ramond-Ramond fields: a 2-form $F$ and a zeroform $M$ dual to the ten-form associated with D8-branes. Note, non-zero value of $M$ leads to the ten-dimensional theory with a cosmological constant [23], the so-called massive Type IIA supergravity [24]. Furthermore, one can consider compactifications where background 
fields $F$ and $G$ have two indices in the noncompact directions. In what follows it will be convenient, instead, to introduce the dual eight-form $\check{F}^{(8)}$ and the six-form $\check{G}^{(6)}$ which have only internal space-time indices. All these Ramond-Ramond field strengths can be combined into a formal sum:

$$
\mathcal{F}=M+i F+G+i \check{G}^{(6)}+\check{F}^{(8)},
$$

so that $\mathcal{F} \in H^{*}(X)$. We denote by $\mathcal{V}_{k}\left(\right.$ resp. $\nu_{k}$ ) two-dimensional superfields (resp. their scalar components) representing different choices of the flux $\mathcal{F}$.

In general, non-zero Ramond-Ramond fluxes break supersymmetry generating an effective superpotential $W\left(\Phi_{i}, \Sigma_{j}, \mathcal{V}_{k}\right)$ in the two-dimensional theory [19,25. Usually it is very difficult to see the superfields $\mathcal{V}_{k}$ explicitly, so we mainly consider the chiral superpotential $W\left(\Phi_{i}\right)$ and the twisted chiral superpotential $\widetilde{W}\left(\Sigma_{j}\right)$ obtained via integrating over the fields $\mathcal{V}_{k}$. A simple way to find effective superpotential is to interpret BPS solitons in $\mathcal{N}=(2,2)$ theory as D-branes wrapped over supersymmetric cycles in $X$.

Let us start with a simple example which is actually a precursor of calibrated geometry. Consider compactification of Type IIA theory on $X$ with a non-zero flux of the 4-form field strength $G$. The effective superpotential looks like [19]:

$$
W\left(\Phi_{i}\right)=\frac{1}{2 \pi} \int_{X} \Omega \wedge G
$$

To see how the formula (2.4) comes about, let us take a D4-brane wrapped over a supersymmetric four-cycle $S \in H_{4}(X, \mathbb{Z})$. In the two-dimensional effective field theory this state is a BPS soliton interpolating between two supersymmetric vacua. Since $G$ jumps across the D4-brane, these vacua correspond to the different four-form fluxes $G_{1}$ and $G_{2}$, such that $\Delta G / 2 \pi=\left(G_{1}-G_{2}\right) / 2 \pi$ is Poincaré dual to the homology class $[S]$. In order to find the superpotential we note that in the effective $\mathcal{N}=(2,2)$ theory the mass of the BPS soliton connecting the two vacua is given by the absolute value of $\Delta W$. On the other hand, the mass of this soliton is given by the mass of the D4-brane wrapped over the special Lagrangian cycle $S$. Therefore we find $\Delta W=\int_{S} \Omega=\frac{1}{2 \pi} \int_{X} \Omega \wedge \Delta G$.

Regarded as a chiral primary state in the Hilbert space of the $\mathcal{N}=2 \mathrm{SCFT}$ associated with the Calabi-Yau space $X, \Delta W=\int_{S} \Omega$ has the mirror non-chiral counterpart [26]:

$$
\Delta \widetilde{W}\left(\Sigma_{j}\right)=\frac{1}{2 \pi} \int_{\widetilde{X}} e^{\widetilde{\mathcal{K}}} \wedge \Delta \mathcal{F}
$$


where $\widetilde{\mathcal{K}}$ is a complexified Kähler class of the mirror Calabi-Yau manifold $\widetilde{X}$. In the dual two-dimensional effective theory obtained by compactification on $\widetilde{X}$, it is natural to interpret (2.5) as a change of twisted superpotential. Therefore, by mirror symmetry we expect the following twisted chiral superpotential:

$$
\widetilde{W}\left(\Sigma_{j}\right)=\frac{1}{2 \pi} \int_{X} e^{\mathcal{K}} \wedge \mathcal{F}
$$

in Type IIA compactification on a Calabi-Yau four-fold $X$.

There are several terms in (2.6) which also can be deduced from the relation between solitons and D-branes. Consider a soliton constructed from a D8-brane wrapped over the entire $X$. Since the value of $M$ changes by $2 \pi$ in crossing the D8-brane, the soliton connects two vacua with different values of the field $M$. In the theory with four supercharges the mass of this soliton $\operatorname{Vol}(X)=\frac{1}{4 !} \int_{X} \mathcal{K} \wedge \mathcal{K} \wedge \mathcal{K} \wedge \mathcal{K}$ is expected to be proportional to the change in the superpotential $\Delta \widetilde{W}$. This is indeed the case, in accordance with the formula (2.6) predicted by the mirror symmetry. In order to obtain another term in (2.6) proportional to the eight-form flux one has to consider a soliton constructed from a D0brane. Since the latter does not wrap any cycle in the Calabi-Yau space $X$, the mass of this soliton does not depend on the moduli of $X$, in accordance with $\Delta \widetilde{W}=\frac{1}{2 \pi} \int_{X} \check{F}^{(8)}$.

The induced superpotential lifts (part of) the classical vacua in the effective twodimensional theory. Now a point in the moduli space $\mathcal{M}_{c}(X) \times \mathcal{M}_{\mathcal{K}}(X)$ corresponds to a supersymmetric vacuum only if it is a minimum of the effective superpotential. This picture suggests to interpret the extremality conditions of the induced superpotential as the supersymmetry conditions in Type IIA theory on the Calabi-Yau space $X$ with RamondRamond fluxes. Let us illustrate this interpretation with the following example. Since Type IIA string theory is related to M-theory via compactification on a circle we expect Type IIA supersymmetry conditions in part to be similar to those in eleven-dimensions [19,27. For instance, if the 4-form $G$ is the only non-vanishing Ramond-Ramond field the supersymmetry conditions should be exactly the same as in M-theory. When $G$ has only internal space-time indices, eq. (2.6) leads to a simple expression for the superpotential proposed in [19]:

$$
\widetilde{W}=\frac{1}{4 \pi} \int_{X} \mathcal{K} \wedge \mathcal{K} \wedge G
$$

Its variation is equivalent to the primitivity condition $G \wedge \mathcal{K}=0$ which is one of the supersymmetry conditions found in [27]. The other supersymmetry constraints require $G^{(3,1)}=0$. This is precisely what one finds from the variation of the superpotential (2.4). 
More generally, we expect the supersymmetry conditions in Type IIA theory on $X$ with Ramond-Ramond fluxes to be equivalent to the supersymmetry conditions in the two-dimensional effective field theory. A supersymmetric vacuum of the two-dimensional theory corresponds to a minimum of the superpotential:

$$
\frac{D W}{D \phi_{i}}=0 \quad \text { and } \quad \frac{D \widetilde{W}}{D \sigma_{i}}=0
$$

where we use the appropriate covariant derivatives $D_{\phi_{i}}=D / D \phi_{i}$ and $D_{\sigma_{j}}=D / D \sigma_{j}$. The form of the covariant connection is determined by the requirement that $D W$ transforms covariantly under a Kähler transformation $K \rightarrow K+F+\bar{F}$, where $F$ is an arbitrary holomorphic function. Since the superpotential transforms as $W \rightarrow e^{-F} W$, we must define the holomorphic connection as $D W=\partial W+(\partial K) W$ in order to have $D W \rightarrow e^{-F} D W$ under the Kähler transformation [28]. In plain english, this means that in supergravity theory with four supercharges a superpotential is a section of a holomorphic line bundle, rather than a usual function. Therefore, the chiral superpotential (2.4) proportional to the choice of $\Omega$ (which is a section of a line bundle over $\mathcal{M}_{c}(X)$ ) exhibits exactly the right behavior.

In a supersymmetric vacuum either $W$ or $\widetilde{W}$ should vanish 2 and, moreover, all the low-energy multiplets must be massive in a vacuum with non-zero superpotential [19]. The value of $W$ (resp. $\widetilde{W}$ ) in the minimum determines the mass of the gravitino fields [20,29]. Therefore, in general we expect that compactifications of massive Type IIA supergravity on a Calabi-Yau four-fold $X$ lead to two-dimensional vacua with negative cosmological constant given by:

$$
\Lambda=-|W|^{2}
$$

Actually, more careful analysis shows that these vacua typically have non-constant dilaton field in the background [30].

2 A simple way to see this using techniques of the present section is to consider a composite domain wall that interpolates between this vacuum and a vacuum with no flux, $\mathcal{F}=0$. Since the values of both $W$ and $\widetilde{W}$ should jump across this domain wall, from the general relation between fluxes and corresponding D-branes it follows that the composite domain wall must be constructed from two kinds of D-branes - D-branes wrapped over special Lagrangian cycles, and D-branes wrapped over holomorphic cycles. Each of these D-branes is BPS since it is wrapped on a supersymmetric cycle. However, the two kinds of D-branes preserve different supersymmetry, so that in total they break supersymmetry completely. 
One can directly check that ten-dimensional supersymmetry conditions are equivalent to the equations (2.8) and (2.9), with the holomorphic functions $W$ and $\widetilde{W}$ given by (2.4) and (2.6). For example, extremizing (2.4) and (2.6) in flat space we find the following simple equations 3 :

$$
G^{(3,1)}=G^{(1,3)}=0
$$

and

$$
\frac{M}{6} \mathcal{K} \wedge \mathcal{K} \wedge \mathcal{K}+\frac{i}{2} F \wedge \mathcal{K} \wedge \mathcal{K}+G \wedge \mathcal{K}+i \check{G}^{(6)}=0
$$

which are precisely the supersymmetry conditions that one finds in compactification of massive Type IIA supergravity on $\mathbf{R}^{2} \times X$ with background Ramond-Ramond fluxes. In a vacuum with non-zero cosmological constant supersymmetry conditions are more subtle due to the dependence on Kähler potential.

Let us now see what kind of corrections we could expect to the semi-classical expressions (2.4) and (2.6). Because the Type IIA dilaton comes in the real gravitational multiplet there are no stringy corrections to the superpotential. Furthermore, the chiral superpotential $W\left(\Phi_{i}\right)$ is exact at the tree level simply because there are no three-branes in Type IIA string theory. On the other hand, the twisted chiral superpotential $\widetilde{W}\left(\Sigma_{j}\right)$ is modified by world-sheet instantons [25] as well as by five-brane instantons [33]. Since a five-brane wrapped over a cycle $S \in H_{6}(X, \mathbb{Z})$ implies the cohomology class of the 4form field $G$ restricted to $S$ to be trivial, non-zero 4-flux can prevent five-brane instanton corrections. Nevertheless, one can use the mirror symmetry to find the exact form of the twisted chiral superpotential [25]. The basic idea is that effective two-dimensional theories constructed by considering Type IIA theory on the mirror Calabi-Yau four-folds $X$ and $\widetilde{X}$ must be equivalent. In particular, we expect:

$$
W_{X}\left(\Phi_{i}\right)=\widetilde{W}_{\widetilde{X}}\left(\Sigma_{i}\right)
$$

Therefore, the world-sheet instanton effects (on the right-hand side of the formula (2.12)) can be found evaluating the period integral (2.4) on the left-hand side of (2.12).

3 Notice, the holomorphic covariant derivative $D_{\phi_{i}} W=\partial_{\phi_{i}} W+\left(\partial_{\phi_{i}} K_{c}\left(\phi_{i}\right)\right) W$ computed using the Kähler potential (2.2) has a nice property that $D_{\phi_{i}} \Omega$ is orthogonal to $\Omega$ [31, 32]. Therefore, one can equivalently define $D_{\phi_{i}}$ as a covariant connection which satisfies $\int_{X}\left(D_{\phi_{i}} \Omega\right) \wedge \bar{\Omega}=0$. 


\section{Solitons and Calibrations}

In general, we can consider a $D(2 p)$-brane of Type IIA theory wrapped over a $2 p$ cycle $S \in H_{2 p}(X, \mathbb{Z})$. In the effective two-dimensional field theory this state looks like a soliton connecting two vacua corresponding to the different set of Ramond-Ramond fluxes on the eight-manifold $X$. The difference between the fluxes is given by the Poincaré dual cohomology class $\Delta \mathcal{F}=\widehat{[S]} \in H^{8-2 p}(X, \mathbb{Z})$.

Furthermore, BPS solitons correspond to D-branes wrapped over supersymmetric cycles. Out of the states with a given set of charges, a soliton that saturates the Bogomolnyi bound has the least mass. In string theory it means that the corresponding D-brane is wrapped over a cycle which has minimal area in its homology class. This property of BPS states constructed from wrapped D-branes has a very nice geometric interpretation in terms of calibrations [1].

Let us remind the definition of calibrated submanifolds, as in Harvey and Lawson [1]. Let $\Psi$ be a closed $k$-form on $X$. We say that $\Psi$ is a calibration if it is less than or equal to the volume on each oriented $k$-dimensional submanifold $S \subset X$. Namely, combining the

orientation of $S$ with the restriction of the Riemann metric on $X$ to the subspace $S$, we can define a natural volume form $\operatorname{vol}\left(T_{x} S\right)$ on the tangent space $T_{x} S$ for each point $x \in S$. Then, $\left.\Psi\right|_{T_{x} S}=\alpha \cdot \operatorname{vol}\left(T_{x} S\right)$ for some $\alpha \in \mathbb{R}$, and we write:

$$
\left.\Psi\right|_{T_{x} S} \leq \operatorname{vol}\left(T_{x} S\right)
$$

if $\alpha \leq 1$. If equality holds for all points $x \in S$, then $S$ is called a calibrated submanifold with respect to the calibration $\Psi$. According to this definition, the volume of a calibrated submanifold $S$ can be expressed in terms of $\Psi$ as:

$$
\operatorname{Vol}(S)=\left.\int_{x \in S} \Psi\right|_{T_{x} S}=\int_{S} \Psi=\int_{X} \Psi \wedge \widehat{[S]}
$$

Since the right-hand side depends only on the cohomology class, we can write:

$$
\operatorname{Vol}(S)=\int_{X} \Psi \wedge \widehat{[S]}=\int_{X} \Psi \wedge \widehat{\left[S^{\prime}\right]}=\left.\int_{x \in S^{\prime}} \Psi\right|_{T_{x} S^{\prime}} \leq \int_{x \in S^{\prime}} \operatorname{vol}\left(T_{x} S^{\prime}\right)=\operatorname{Vol}\left(S^{\prime}\right)
$$

for any other submanifold $S^{\prime}$ in the same homology class. Therefore, we have just demonstrated that calibrated manifolds have minimal area in their homology class, the property we expect of the wrapped D-branes to represent BPS solitons. 
From the supersymmetry algebra it follows that the mass of a BPS soliton is equal to the change in superpotential, $|\Delta W|$. Since the former is given by the formula (3.1) and the change of the Ramond-Ramond fluxes $\Delta \mathcal{F}$ is Poincaré dual to the homology class $[S]$, we obtain the following general formula for the superpotential:

$$
W\left(\Phi_{i}, \Sigma_{j}\right)=\frac{1}{2 \pi} \int_{X} \Psi \wedge \mathcal{F}
$$

Calibrations are very useful in the study of manifolds with special holonomy groups. This is because special holonomy groups are characterized by the existence of covariantly constant forms. These forms can be used as calibrations. Let us now illustrate the formula (3.2) with some examples of special calibrations.

$S U(4)$ Calibrations: The main goal of this paper is to study Type IIA compactifications on Calabi-Yau four-folds. These manifolds have $S U(4)$ holonomy group. There are two types of calibrations on manifolds with $S U(4)$ holonomy group (more generally, on manifolds with $S U(n)$ holonomy group). If we take:

$$
\Psi=\frac{1}{2} \mathcal{K}^{2}+\operatorname{Re}\left(e^{i \theta} \Omega\right)
$$

we obtain a $U(1)$ family of the so-called Cayley calibrations. Substituting the Cayley calibration into (3.2), we reproduce the chiral superpotential (2.4) plus the twisted chiral superpotential (2.7). This is, of course, not surprising since deriving the formula (2.4) we implicitly used the special case of the calibration (3.3) corresponding to special Lagrangian submanifolds. Indeed, a special Lagrangian manifold is a calibrated submanifold with respect to the special Lagrangian calibration:

$$
\Psi=\operatorname{Re}(\Omega)
$$

One can obtain the other type of $S U(4)$ calibrations - Kähler calibrations — considering various powers of the complexified Kähler form:

$$
\Psi=\frac{1}{p !} \mathcal{K}^{p}
$$

Since $\mathcal{K}$ is covariantly constant, (3.4) is covariantly constant as well. The submanifolds calibrated by this $\Psi$ are complex submanifolds $S \subset X$ of complex dimension $p$. It is easy to see that in this case the general formula (3.2) yields the twisted chiral superpotential (2.6) predicted by mirror symmetry, with the right numerical coefficients. 
$G_{2}$ Calibrations: In our derivation of the superpotential (3.2) we made very mild assumptions about the geometry of the manifold $X$. Namely, $X$ had to be a Riemannian manifold, not necessarily eight-dimensional, such that a compactification of Type IIA string on $X$ preserved four unbroken supersymmetries. Hence, all of the above arguments also apply verbatim to the seven-dimensional manifolds of $G_{2}$ holonomy, with the word 'a soliton' replaced by 'a domain wall'. This change is due to the fact that now we talk about $\mathcal{N}=2$ three-dimensional effective field theory, so that different vacua are connected by domain walls rather than by solitons.

Manifolds of $G_{2}$ holonomy are characterized by a covariantly constant three-form $\Psi^{(3)}$ invariant under the exceptional group $G_{2}$. From the formula (3.2) we expect to find the following superpotential in the effective three-dimensional theory:

$$
W=\frac{1}{2 \pi} \int_{X} \Psi^{(3)} \wedge G
$$

A domain wall that connects different vacua in this theory corresponds to a $D 4$-brane wrapped over three-dimensional associative submanifold $S$ calibrated by $\Psi^{(3)}$.

The coassociative calibration $\Psi^{(4)}=\star \Psi^{(3)}$ is defined as a 4 -form Hodge dual to $\Psi^{(3)}$. The submanifolds calibrated by $\Psi^{(4)}$ are four-dimensional coassociative submanifolds. Since a domain wall in three non-compact dimensions can be constructed from an NS5-brane wrapped over a coassociative cycle $S$, from the expression (3.2) we expect the following superpotential corresponding to the calibration $\Psi^{(4)}$ :

$$
W=\frac{1}{2 \pi} \int_{X} \Psi^{(4)} \wedge H
$$

Here $H$ is the NS-NS three-form field strength.

Spin(7) Calibrations: Even though our arguments do not directly apply to the case of eight-manifolds with $\operatorname{Spin}(7)$ holonomy which break too much supersymmetry, it is amusing to employ the general formula (3.2) to the Cayley calibration $\Psi^{(+)}$of degree four. The form $\Psi^{(+)}$is self-dual, $\Psi^{(+)}=\star \Psi^{(+)}$. We conjecture the following expression for the superpotential:

$$
W=\frac{1}{2 \pi} \int_{X} \Psi^{(+)} \wedge G
$$

In the present paper we will not pursue the proof of the formulas (3.5) - (3.7) for the manifolds of the exceptional holonomy.

As one can definitely see, calibrated geometries prove to be very useful in writing superpotentials induced by background fluxes. 


\section{A Mathematical Application: Counting BPS Solitons}

To explain the geometric meaning of the effective superpotentials found in the previous sections, we recall that the expectation values of the fields $\phi_{i}$ and $\sigma_{j}$ play the role of the Kähler and the complex moduli of a Calabi-Yau space $X$, respectively. Therefore, in a background with zero Ramond-Ramond fields the classical space of supersymmetric vacua of the two-dimensional effective field theory is simply the moduli space of $X$.

When Ramond-Ramond fluxes do not vanish, the degeneracy of supersymmetric ground states is (partly) lifted by the induced superpotential $6 W\left(\Phi_{i}, \Sigma_{j}, \mathcal{V}_{k}\right)$. Solving the equations:

$$
\frac{D W}{D \nu_{k}}=0
$$

for $\nu_{k}$, one can integrate out the fields $\mathcal{V}_{k}$ and obtain the effective chiral superpotential $W\left(\Phi_{i}\right)$ and the twisted chiral superpotential $\widetilde{W}\left(\Sigma_{j}\right)$. If for certain values of $\phi_{i}$ and $\sigma_{j}$ the resulting superpotentials have a minimum, then the two-dimensional field theory has a supersymmetric vacuum obtained from the supersymmetric compactification on $X$ with the Kähler and the complex moduli defined by the solution of (2.8). Thus, supersymmetric vacua form a subspace in the moduli space of $X$, as shown in Fig.1. For example, a compactification with a $G$-flux is supersymmetric only if the Kähler structure of $X$ is such that $G$ is primitive [27]. This condition is equivalent to (2.8) with the twisted superpotential (2.7).

Notice that the superpotentials (2.4) and (2.6) constructed in the previous section depend only on the cohomology class of the Ramond-Ramond flux $\mathcal{F}$. Therefore, when (4.1) has several solutions, a minimum of the effective superpotential with the fields $\mathcal{V}_{k}$ integrated out corresponds to multiple supersymmetric vacua, all representing the same cohomology class $[\mathcal{F}]$. This multiplicity appears, for example, in a computation of the Witten index in the effective two-dimensional theory. For every point $\left(\phi_{i}, \sigma_{j}\right)$ in the moduli space of $X$, let us denote its contribution to the supersymmetric index by $\mathcal{I}_{[\mathcal{F}]}(X)$ :

$$
\mathcal{I}_{[\mathcal{F}]}(X)=\operatorname{Tr}(-1)^{F}
$$

The right-hand side of this definition is the supersymmetric index in the two-dimensional effective theory with the dynamical fields $\nu_{k}$ and the Calabi-Yau moduli (fields $\left\{\phi_{i}\right\}$ and

4 For the purposes of the present section we restore dependence on the moduli $\nu_{k}$ of the Ramond-Ramond fields. Since in many cases the explicit form of the complete superpotential $W\left(\Phi_{i}, \Sigma_{j}, \mathcal{V}_{k}\right)$ is not known, we do not differentiate between chiral and twisted chiral superfields. 


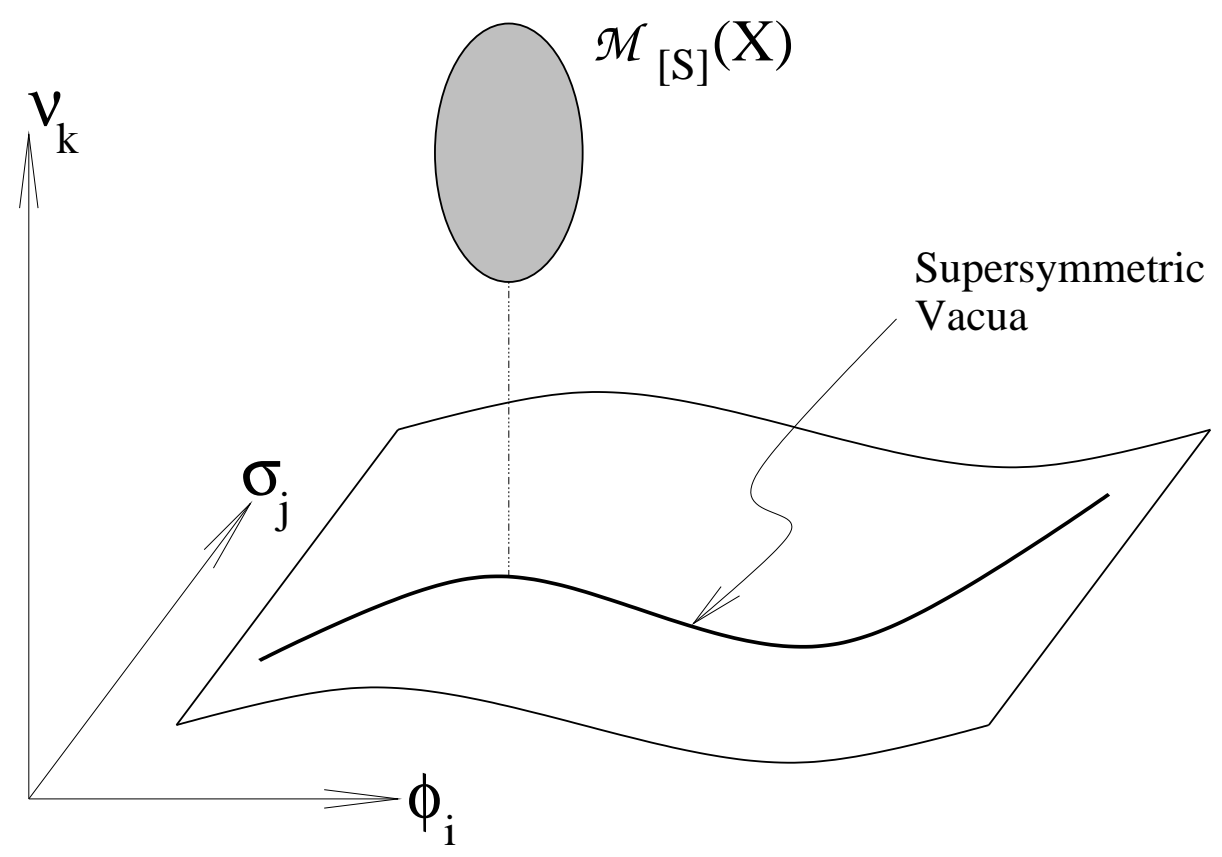

Fig. 1: Classical vacua of the effective two-dimensional field theory form a subspace in the moduli space of $X$. Every point of this subspace is associated with the moduli space of a supersymmetric cycle $S$, for a given complex and Kähler structure of $X$.

$\left.\left\{\sigma_{j}\right\}\right)$ being fixed. The latter must satisfy the supersymmetry conditions (2.8) in order for $\mathcal{I}_{[\mathcal{F}]}(X)$ to be non-zero. Since we regard $\phi_{i}$ and $\sigma_{j}$ as background fields, the supersymmetric index (which in simple cases counts the number of supersymmetric ground states in the effective theory) is expected to be stable under deformations of the four-fold $X$ [34]. Therefore, $\mathcal{I}_{[\mathcal{F}]}(X)$ must be a topological invariant of $X$, at least as long as we do not cross a surface of marginal stability ${ }^{5}$ in the moduli space of $X$. For a given $X$ and $[\mathcal{F}] \in H^{*}(X, \mathbb{Z})$, let $\mathcal{M}_{[\mathcal{F}]}(X)$ be the set of solutions to (4.1). Then $\mathcal{I}_{[\mathcal{F}]}(X)$ is equal to the Euler number of $\mathcal{M}_{[\mathcal{F}]}(X)$ :

$$
\mathcal{I}_{[\mathcal{F}]}(X)=\chi\left(\mathcal{M}_{[\mathcal{F}]}(X)\right)
$$

There is an equivalent geometric definition of $\mathcal{I}$ in terms of the homology class $[S]$ Poincaré dual to $[\mathcal{F}]$. With every supersymmetric vacuum representing the RamondRamond flux $\mathcal{F}$ we can associate a BPS soliton constructed from a D-brane 6 wrapped

5 The meaning of this term will become clear in a moment when we give a 'dual' definition of the invariants $\mathcal{I}(X)$ in terms of supersymmetric cycles in $X$.

6 Due to the anomaly inflow [35], D-branes on curved manifolds carry induced charge of lower 
over the dual supersymmetric cycle $S$. Hence, $\mathcal{I}$ can be defined as the "number" of supersymmetric cycles with the homology class $[S]$. More precisely, a system of invariants $\mathcal{I}$ for a Calabi-Yau four-fold $X$ is a family of maps $\mathcal{I}: H_{*}(X, \mathbb{Z}) \rightarrow \mathbb{Z}$ defined by:

$$
\mathcal{I}_{[S]}(X)=\chi\left(\mathcal{M}_{[S]}(X)\right)
$$

where $\mathcal{M}_{[S]}(X)$ is the moduli space of calibrated submanifolds together with flat line bundles. It is important to bear in mind that a D-brane carries a $U(1)$ gauge field. We do not discuss here the important questions of smoothness and compactness, simply assuming that there is a compactification of $\mathcal{M}_{[S]}(X)$ such that (4.4) makes sense. The motivation for this assumption is based in part on the following two examples where a suitable compactification can be achieved.

When $[S]$ is an element of $H_{2}(X, \mathbb{Z})$, the supersymmetric cycles are given by the submanifolds calibrated with respect to the Kähler form of $X$. Therefore, in this case the invariant $\mathcal{I}_{[S]}(X)$ is equal to the Euler number of the moduli space of holomorphic curves with a flat line bundle. Unlike the definition of the Gromov-Witten invariants [36, 37, there are no constraints on $S$ except to stay within a given homology class. Therefore one might worry that there are infinitely many contributions from curves of arbitrary topology. However, by the Riemann-Roch theorem the virtual (complex) dimension of the moduli space of genus $g$ curves in a Calabi-Yau four-fold is equal to $(1-g)$, so that generic point in $\mathcal{M}_{[S]}(X)$ corresponds to a rational homology 2-sphere. For such curves dimension of the space of flat $U(1)$ connections is zero. At least for some simple cases, one may hope to use the close relation to the Gromov-Witten invariants to compute $\mathcal{I}_{[S]}(X)$.

Although it is difficult to count supersymmetric cycles in general case, we can predict the asymptotic growth of $\mathcal{I}_{[S]}(X)$ under rescaling $[S] \rightarrow N[S]$ by a large number $N$. To estimate the "number" of holomorphic curves in a Calabi-Yau four-fold $X$ we use the microscopic interpretation of black hole entropy [38]. The analysis is very similar to the derivation of the entropy of extreme black holes constructed from Calabi-Yau three-folds [39,40]. Consider M-theory compactification on a four-fold $X$ down to three dimensions. Then, an M2-brane wrapped around a 2-cycle in the homology class $[S] \in H_{2}(X, \mathbb{Z})$

dimensional branes. Therefore, the accurate relation between $\mathcal{I}_{[\mathcal{F}]}(X)$ and the invariant $\mathcal{I}_{[S]}(X)$ defined below involves bound states of D-branes. This subtlety, however, is not important for the definition of $\mathcal{I}_{[S]}(X)$ unless we are interested in the precise relation to $\mathcal{I}_{[\mathcal{F}]}(X)$. 
represents the extreme BTZ black hole [41] with charge [S]. The entropy of this black hole is related to the number of BPS states with a given charge:

$$
S_{B T Z}=\log \mathcal{I}_{[S]}(X)
$$

On the other hand, Bekenstein-Hawking entropy of the extreme BTZ black hole is related to its mass $M_{B T Z}$ [42]:

$$
S_{B T Z} \sim \sqrt{M_{B T Z}}
$$

In $\mathrm{M}$ theory the mass $M_{B T Z}$ is given by the mass of a membrane wrapped around a cycle $S$ of minimal area, so that as $[S] \rightarrow N[S]$ the mass scales as $M_{B T Z} \sim N$. Therefore, from (4.6) we conclude that to the leading order in $N \gg 1$ the number of BPS states grows as:

$$
\mathcal{I}_{[S]}(X) \sim \exp (a \sqrt{N})
$$

for some constant $a$. There is one remark in place here. The above argument based on the black hole entropy formula actually predicts the growth of cohomologies of $\mathcal{M}_{[S]}(X)$, rather than its Euler number $\chi\left(\mathcal{M}_{[S]}(X)\right)$. The latter should have the same large $N$ behavior unless there is a nearly perfect cancellation between cohomologies of odd and even degree. This is indeed the case for Calabi-Yau three-folds [40]. If such a miracle also happens for Calabi-Yau four-folds, then the formula (4.7) should be understood only as an upper bound. It says that $\mathcal{I}_{[S]}(X)$ grow not faster than $\exp (a \sqrt{N})$.

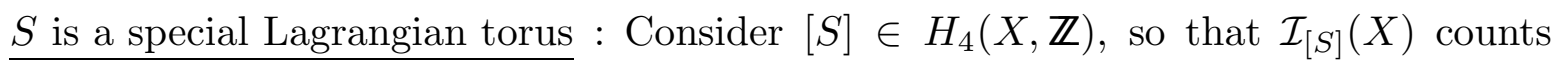
special Lagrangian submanifolds in the homology class $[S]$ with a choice of flat $U(1)$ connection. A similar invariant counting special Lagrangian homology 3 -spheres in Calabi-Yau three-folds was recently introduced by Joyce [14]. According to [43, 13], the mirror symmetry can be understood as an equivalence between derived categories over the mirror spaces $X$ and $\widetilde{X}$. In particular, it means that the moduli space of special Lagrangian manifolds (with a flat connection) in a given homology class $[S] \in H_{4}(X)$ is equivalent to the moduli space, $\mathcal{M}\left(V_{c h}, \widetilde{X}\right)$, of stable vector bundles over the mirror space $\widetilde{X}$, where the Chern class $c h \in H^{*}(\widetilde{X})$ is determined by $[S]$. Therefore, when $S$ is a special Lagrangian manifold, eq. (4.4) can be written as:

$$
\mathcal{I}_{[S]}(X)=\chi\left(\mathcal{M}\left(V_{c h}, \widetilde{X}\right)\right)
$$

Now suppose that $X$ admits a special Lagrangian 4-torus fibration [12, 44], and let us take $[S]$ to be the class of the fiber. Then, according to the conjecture of Strominger, 
Yau and Zaslow [12], there is a compactification of $\mathcal{M}_{[S]}(X)$ isomorphic to the mirror Calabi-Yau manifold:

$$
\mathcal{M}_{[S]}(X) \cong \widetilde{X}
$$

In this case we have $\mathcal{I}_{[S]}(X)=\chi(\tilde{X})=\chi(X)$. Obviously, the latter is a topological invariant of $X$, though not a new one.

\section{New Supersymmetric Vacua}

In the previous sections we found a set of constraints on Calabi-Yau moduli and Ramond-Ramond fluxes which preserve $\mathcal{N}=2$ supersymmetry in two dimensions. There is a compact way of writing these conditions in terms of the effective superpotentials (2.4) and (2.6).

From the above discussion it is also clear that these supersymmetry constraints are highly restrictive, so that usually it is very difficult to find an explicit solution. For example, when $G$ is the only non-zero field one has to look for integral primitive forms of Hodge type $(2,2)$. This problem was studied in a recent work [18].

Below we present a family of solutions to the supersymmetry conditions with non-zero fluxes of $M, G$, and $\check{F}^{(8)}$. For the sake of simplicity, we assume that the two-form flux $F$ and the six-form flux $\check{G}^{(6)}$ vanish, so that superpotential is real.

Another insight from the supersymmetry condition (2.11) is that different components of Ramond-Ramond fields which transform differently under the action of the $S U(4)$ holonomy group must vanish separately. Indeed, by definition, a wedge product with the Kähler form $\mathcal{K}$ takes one Ramond-Ramond form to another form (of degree greater by 2 ) from the same irreducible representation. Since the 0-form $M$ and the 8-form $\check{F}^{(8)}$ are singlets under $S U(4)$, their contribution in the effective superpotential can be canceled also only by singlet component of the $G$-flux. Such fields can be written as wedge products of the Kähler form $\mathcal{K}$ :

$$
\frac{M}{2 \pi}=n_{0}, \quad \frac{G}{2 \pi}=\frac{n_{4}}{2} \mathcal{K} \wedge \mathcal{K}, \quad \frac{\check{F}^{(8)}}{2 \pi}=\frac{n_{8}}{4 !} \mathcal{K} \wedge \mathcal{K} \wedge \mathcal{K} \wedge \mathcal{K}
$$

Of course, to consort with the flux quantization of the field $F$ one has to make an appropriate choice of the Kähler structure, such that $\mathcal{K} \in H^{2}(X, \mathbb{Z})$ and $n_{8}$ is an integer number. The quantization condition of the field $G$ is more subtle [22]:

$$
\frac{G}{2 \pi}-\frac{p_{1}(X)}{4} \in H^{2}(X, \mathbb{Z})
$$


When $\chi(X) / 24 \in \mathbb{Z}$, the number $n_{4}$ is integer. In massive Type IIA string theory $\frac{M}{2 \pi}=n_{0}$ also has to be an integer.

The ansatz (5.1) automatically satisfies the supersymmetry condition (2.10) in flat two-dimensional space. Therefore, it remains to check the conditions that follow from the twisted chiral superpotential (2.6):

$$
\int_{X} \frac{M}{4 !} \mathcal{K} \wedge \mathcal{K} \wedge \mathcal{K} \wedge \mathcal{K}+\frac{1}{2 !} G \wedge \mathcal{K} \wedge \mathcal{K}+\check{F}^{(8)}=0
$$

and

$$
\frac{M}{6} \mathcal{K} \wedge \mathcal{K} \wedge \mathcal{K}+G \wedge \mathcal{K}=0
$$

Substituting the ansatz (5.1) into (5.2) and (5.3) we obtain a system of two algebraic equations:

$$
n_{0}+6 n_{4}+n_{8}=0, \quad \text { and } \quad n_{0}+3 n_{4}=0
$$

which must be solved in integer numbers. From the second equation we see that there are

no solutions when $n_{4} \in \mathbb{Z}+\frac{1}{2}$. Hence, $\chi(X)$ has to be divisible by 24 , so that $n_{4}$ is an integer. Then, the solution is given by:

$$
n_{0}=n_{8}=-3 n_{4}
$$

This solution can be easily generalized to include the Ramond-Ramond two-form and six-form fluxes on the Calabi-Yau four-fold.

\section{Appendix I. Massive Type IIA Supergravity on Eight-Manifolds}

In this appendix we present a microscopic test of our results. In particular, we consider compactification of Type IIA string theory on a Calabi-Yau four-fold $X$, and ask for the conditions on the background Ramond-Ramond fields to preserve at least $\mathcal{N}=2$ supersymmetry in two dimensions. We follow the notations of [19,27] where a similar analysis was carried out for compactifications with a $G$-field.

In the large volume limit the effective superstring dynamics is described by a supergravity theory, viz. massive Type IIA supergravity [24], since the 0-form field $M$ is generically non-zero. The bosonic field content of the massive Type IIA supergravity contains the metric $g_{M N}$, the dilaton $\varphi$, a vector field $A_{M}$, tensor fields $B_{M N}$ and $C_{M N P}$ and 
a mass parameter $M$. By supersymmetry we also have non-chiral spinor fields: a gravitino $\psi_{M}$ and a dilatino $\lambda$. The bosonic part of the Lagrangian looks like [24]:

$$
\begin{gathered}
L=\sqrt{-g}\left[-\frac{1}{4} R+\frac{1}{2}\left(\nabla_{M} \varphi\right)\left(\nabla^{M} \varphi\right)+\frac{1}{12} e^{-\varphi} H_{M N P} H^{M N P}+\right. \\
\left.+\frac{1}{48} e^{\frac{1}{2} \varphi} G_{M N P Q} G^{M N P Q}+\frac{1}{4} M^{2} e^{\frac{3}{2} \varphi} B_{M N} B^{M N}+\frac{1}{8} M^{2} e^{\frac{5}{2} \varphi}+\ldots\right]
\end{gathered}
$$

where we introduced the gauge-invariant field strengths 45:

$$
\begin{gathered}
F_{M N}=2 \partial_{[M} A_{N]}+M B_{M N} \\
H_{M N P}=3 \partial_{[M} B_{N P]} \\
G_{M N P Q}=4 \partial_{[M} C_{N P Q]}+24 B_{[M N} \partial_{P} A_{Q]}+6 M^{2} B_{[M N} B_{P Q]}
\end{gathered}
$$

Note that our notations slightly differ from the notations in [24]. One reason for this is to simplify the comparison with the ordinary Type IIA supergravity. In the limit $M \rightarrow 0$ the last two terms in (II.1) disappear and we end up with the standard effective action for the massless Type IIA fields. When $M \neq 0$, we can gauge away the vector field $A_{M}$ by gauge transformations of $B_{M N}$, leaving the latter with a mass but without gauge invariance in a Higgs-type mechanism. The value of the ten-dimensional cosmological constant in the massive phase is also determined by $M$. We use the metric in the Einstein frame which is related to the string metric by the rescaling:

$$
g_{M N}^{\mathrm{st}}=e^{\frac{1}{2} \varphi} g_{M N}
$$

The action of the massive Type IIA supergravity is invariant under 16 left and 16 right supersymmetry transformations, such that the left supersymmetries are chiral while the right supersymmetries are anti-chiral, with the ten-dimensional chirality operator $\Gamma_{11}$. Below we use the explicit form of the supersymmetry transformations only for the fermionic fields. For the gravitino we have:

$$
\begin{gathered}
\delta \psi_{M}=\nabla_{M} \eta-\frac{M}{32} e^{\frac{5}{4} \varphi} \Gamma_{M} \eta-\frac{1}{32} e^{\frac{3}{4} \varphi} F_{N P}\left(\Gamma_{M}{ }^{N P}-14 \delta_{M}^{N} \Gamma^{P}\right) \Gamma_{11} \eta+ \\
+\frac{1}{48} e^{-\frac{1}{2} \varphi} H_{N P Q}\left(\Gamma_{M}^{N P Q}-9 \delta_{M}^{N} \Gamma^{P Q}\right) \Gamma_{11} \eta+\frac{1}{128} e^{\frac{1}{4} \varphi} G_{N P Q R}\left(\Gamma_{M}^{N P Q R}-\frac{20}{3} \delta_{M}^{N} \Gamma^{P Q R}\right) \eta
\end{gathered}
$$

and for the dilatino:

$$
\delta \lambda=-\frac{1}{2 \sqrt{2}}\left(\partial_{M} \varphi\right) \Gamma^{M} \eta-\frac{5}{8 \sqrt{2}} M e^{\frac{5}{4} \varphi} \eta+\frac{3}{8 \sqrt{2}} e^{\frac{3}{4} \varphi} F_{M N} \Gamma^{M N} \Gamma_{11} \eta+
$$




$$
+\frac{1}{12 \sqrt{2}} e^{-\frac{1}{2} \varphi} H_{M N P} \Gamma^{M N P} \Gamma_{11} \eta-\frac{1}{96 \sqrt{2}} e^{\frac{1}{4} \varphi} G_{M N P Q} \Gamma^{M N P Q} \eta
$$

In order to cancel the anomaly (2.3) one has to introduce $N=\frac{1}{24} \chi(X)$ fundamental strings filling two-dimensional non-compact space. We represent these strings by the following maximally symmetric ansatz for the field strength $H_{M N P}$ :

$$
H_{\mu \nu m}=\epsilon_{\mu \nu} \partial_{m} f\left(x^{m}\right)
$$

where the function $f\left(x^{m}\right)$ depends only on the coordinates on $X$. A space-filling fundamental string is invariant under the supersymmetry transformations (1.3) - (I.4) with the supersymmetry parameter $\eta$ satisfying the projection relation:

$$
\Gamma_{01} \Gamma_{11} \eta=\eta
$$

where we used the standard notation $\Gamma_{M_{1} \ldots M_{n}}=\frac{1}{n !} \Gamma_{\left[M_{1}\right.} \ldots \Gamma_{\left.M_{n}\right]}$ for the antisymmetrized product of gamma-matrices. With this choice of sign, the relation (I.6) implies that the supersymmetry is preserved by the spinor $\eta$ which has positive eight-dimensional chirality. In other words, if we decompose $\eta$ as:

$$
\eta=\epsilon \otimes \xi+\epsilon^{*} \otimes \xi^{*}
$$

then the eight-dimensional commuting spinor $\xi$ must satisfy $\gamma_{9} \xi=\xi$, where we also make the $10=2+8$ split of the ten-dimensional gamma-matrices:

$$
\Gamma_{\mu}=\gamma_{\mu} \otimes \gamma_{9}, \quad \Gamma_{m}=1 \otimes \gamma_{m}
$$

The opposite choice of sign in the formula (II.6) would correspond to anti-strings which make a negative contribution to the total charge $N$. Since Calabi-Yau four-folds usually admit only one sort of nowhere-vanishing chiral spinorst, the total number of strings, $N$, must be positive. We choose $\xi$ to be a covariantly constant complex spinor of unit norm. Note, the existence of such a spinor on the Calabi-Yau four-fold $X$ automatically implies $\mathcal{N}=(2,2)$ supersymmetry in two dimensions. Using the spinor $\xi$ we define the complex structure $J_{m}{ }^{n}=i \xi^{\dagger} \gamma_{m}{ }^{n} \xi$ and the Kähler form $\mathcal{K}_{a \bar{b}}=i g_{a \bar{b}}$. Since the metric on $X$ is of

7 Calabi-Yau four-folds that admit spinors of both chiralities have zero Euler number. According to the formula (2.3), no new interesting vacua with non-zero $G$-flux can be found in compactification on such $X$. 
type $(1,1)$, it is convenient to think of 'holomorphic' gamma-matrices $\gamma^{a}$ and $\gamma_{a}$ as creation and annihilation operators:

$$
\gamma^{a} \xi=0, \quad \gamma_{a} \xi^{*}=0, \quad \gamma^{\bar{a}} \xi^{*}=0, \quad \gamma_{\bar{a}} \xi=0
$$

The field $H$ is taken in the form ([.5), while all the fermionic fields are assumed to vanish in the background. As we will see in a moment, a maximally symmetric compactification on $X$ with nontrivial Ramond-Ramond fields typically leads to warped metric:

$$
d s_{10}^{2}=\Delta^{-1}\left(d s_{2}^{2}\left(x^{\mu}\right)+d s_{8}^{2}\left(x^{m}\right)\right)
$$

where we introduced the warp factor $\Delta\left(x^{m}\right)$. For now, both $\Delta\left(x^{m}\right)$ and $f\left(x^{m}\right)$ are scalar functions of the coordinates on $X$. Below we show that these two functions are related by the supersymmetry conditions. Since two-dimensional space-time is assumed to be maximally symmetric (i.e. flat Minkowski space-time, de Sitter space, or anti-de Sitter space) the $\mu$-component of the covariant derivative satisfies the following commutation relation [20]:

$$
\left[\nabla_{\mu}, \nabla_{\nu}\right]=\Lambda \mathcal{X}_{\mu \nu}
$$

where $\Lambda$ is two-dimensional cosmological constant and $\mathcal{X}_{\mu \nu}$ is the Lorentz generator.

Using the proper rescaling of Dirac gamma-matrices:

$$
\Gamma_{M} \quad \rightarrow \quad \Delta^{-1 / 2} \Gamma_{M}, \quad \Gamma^{M} \rightarrow \Delta^{1 / 2} \Gamma^{M}
$$

we rewrite the supersymmetry variations (II.3)-(I.4) of the fermionic fields in the metric ([.9). For the gravitino we get:

$$
\begin{aligned}
\delta \psi_{M}=\nabla_{M} \eta & -\frac{1}{4} \partial_{N}(\log \Delta) \Gamma_{M}{ }^{N} \eta+\frac{\Delta}{48} e^{-\frac{1}{2} \varphi}\left(\Gamma_{M} \not H-12 H_{M P Q} \Gamma^{P Q}\right) \Gamma_{11} \eta- \\
& -\frac{M}{32} \Delta^{-1 / 2} e^{\frac{5}{4} \varphi} \Gamma_{M} \eta-\frac{\Delta^{1 / 2}}{32} e^{\frac{3}{4} \varphi}\left(\Gamma_{M} \not H^{\prime}-16 F_{M P} \Gamma^{P}\right) \Gamma_{11} \eta+ \\
& +\frac{\Delta^{3 / 2}}{128} e^{\frac{1}{4} \varphi}\left(\Gamma_{M} G-\frac{32}{3} G_{M P Q R} \Gamma^{P Q R}\right) \eta
\end{aligned}
$$

And the variation of the dilatino in the metric ([.9) looks like:

$$
\begin{aligned}
\delta \lambda= & -\frac{\Delta^{1 / 2}}{2 \sqrt{2}}\left(\partial_{M} \varphi\right) \Gamma^{M} \eta-\frac{5}{8 \sqrt{2}} M e^{\frac{5}{4} \varphi} \eta+\frac{3}{8 \sqrt{2}} \Delta e^{\frac{3}{4} \varphi} \not \mathbf{\Gamma} \Gamma_{11} \eta+ \\
& +\frac{\Delta^{3 / 2}}{12 \sqrt{2}} e^{-\frac{1}{2} \varphi} \not H \Gamma_{11} \eta-\frac{\Delta^{2}}{96 \sqrt{2}} e^{\frac{1}{4} \varphi} G \eta
\end{aligned}
$$


where we use a short notation $\phi$ for the total contraction $G_{M N P Q} \Gamma^{M N P Q}$, etc.

Now we put the variations of the fermion fields to zero and project the corresponding equations onto subspaces of positive and negative chirality. Let us assume that the spinor $\epsilon$ has positive chirality. The spinor $\epsilon$ of negative chirality leads to the complex conjugated supersymmetry conditions.

We also have to make a decomposition ([.7) of the spinor $\eta$ and assume that the twodimensional Killing spinor $\epsilon$ satisfies an equation like $\nabla_{\mu} \epsilon=\widetilde{m}_{\psi} \gamma_{\mu} \epsilon+m_{\psi} \gamma_{\mu} \epsilon^{*}$, where $m_{\psi}$ (resp. $\widetilde{m}_{\psi}$ ) represents the mass (resp. the twisted mass) of the two-dimensional gravitino field. From the commutation relations (I.10) for the $\nabla_{\mu}$ it follows that either $m_{\psi}$ or $\tilde{m}_{\psi}$ must be equal to zero. Hence, one can distinguish two cases:

i) The cosmological constant is given by $\Lambda=-\left|\widetilde{m}_{\psi}\right|^{2}$ and the Killing spinor $\epsilon$ satisfies:

$$
\nabla_{\mu} \epsilon=\widetilde{m}_{\psi} \gamma_{\mu} \epsilon
$$

ii) The cosmological constant is given by $\Lambda=-\left|m_{\psi}\right|^{2}$ and the Killing spinor $\epsilon$ satisfies:

$$
\nabla_{\mu} \epsilon=m_{\psi} \gamma_{\mu} \epsilon^{*}
$$

The second possibility corresponds to compactification with non-zero value of the $(4,0)$ part of the background $G$-flux. This follows from the $\mu$-component of (I.11) which in this case gives a relation between the warp factor $\Delta$ and the function $f$ :

$$
\left(\partial_{m} \Delta\right)+\frac{1}{54} \Delta^{2} e^{-\frac{1}{2} \varphi}\left(\partial_{m} f\right)=0
$$

and:

$$
m_{\psi} \zeta^{*}-\frac{M}{32} \Delta^{-1 / 2} e^{\frac{5}{4} \varphi} \zeta-\frac{\Delta^{1 / 2}}{16} e^{\frac{3}{4} \varphi} \text { F } \zeta+\frac{\Delta^{3 / 2}}{128} e^{\frac{1}{4} \varphi} \notin \zeta=0
$$

Since the complex spinor $\zeta$ on a Calabi-Yau four-fold obeys the following relation [2]:

$$
\gamma^{a b c d} \zeta=\Omega^{a b c d} \zeta^{*}
$$

from the second equation ([1.16) we get:

$$
m_{\psi}=\frac{\Delta^{3 / 2}}{128} e^{\frac{1}{4} \varphi} G_{a b c d} \Omega^{a b c d}
$$

Integrating over the entire Calabi-Yau four-fold $X$ and using the self-duality of $G^{(0,4)}$, we obtain the formula for the gravitino mass:

$$
m_{\psi}=\frac{1}{l_{2}} \int_{X} G \wedge \Omega
$$


where we introduced two-dimensional scale parameter $l_{2}=128 \int d^{8} x \Delta^{-3 / 2} e^{-\frac{1}{4} \varphi}$. Notice the striking similarity between $(\mathbb{I . 1 8})$ and the formula for the effective superpotential $W \sim$ $\int_{X} G \wedge \Omega$. This is a non-trivial check of the proposed expression (2.4) for the effective superpotential induced by background $G$-flux. Using microscopic arguments, here we found that compactifications with $G^{(0,4)} \neq 0$ lead to two-dimensional vacua where mass for the gravitino field is given by ([.18). In a supersymmetric situation masses of the bosonic modes of the supergravity multiplet should be related to $m_{\psi}$ in a supersymmetric fashion. In particular, one should expect a non-zero cosmological constant $\Lambda=-\left|m_{\psi}\right|^{2}$. However, it turns out that compactification with a $G_{4,0}$ flux alone leads to a solution with zero cosmological constant and, therefore, implies broken supersymmetry [19].

However, Type IIA vacua with non-zero values of $m_{\psi}$ or $\widetilde{m}_{\psi}$ have non-constant dilaton. In fact, multiplying (I.11) by $\Gamma^{M}$ from the left and taking the trace over index ' $M$ ' we obtain the following supersymmetry condition:

$$
\begin{aligned}
& \Gamma^{M} \nabla_{M} \eta-\frac{9}{4} \not \partial(\log \Delta) \eta-\frac{\Delta}{24} e^{-\frac{1}{2} \varphi} \not H \Gamma_{11} \eta- \\
& -\frac{5}{16} M \Delta^{-1 / 2} e^{\frac{5}{4} \varphi} \Gamma_{M} \eta+\frac{3}{16} \Delta^{1 / 2} e^{\frac{3}{4} \varphi} \not \mid \Gamma_{11} \eta+\frac{1}{192} \Delta^{3 / 2} e^{\frac{1}{4} \varphi} \not \eta=0
\end{aligned}
$$

The second line of this equation looks very similar to the variation of the dilatino ([.12). In fact, from the linear combination of $(\underline{[.19})$ and $(\underline{1.12})$ we get:

$$
\Gamma^{M} \nabla_{M} \eta=-\frac{1}{4}(\not \partial \varphi) \eta
$$

Here, for the sake of simplicity, we assumed that $\Delta$ and $f$ are constant 8 . For example, in the gauge we are using, where the Killing spinor $\epsilon$ satisfies (‥13), we obtain:

$$
\left(8 \widetilde{m}_{\psi}+\not \partial \varphi\right) \epsilon=0
$$

This equation implies that:

$$
(\partial \varphi)^{2}=64\left|m_{\psi}\right|^{2}
$$

In the gauge we are using a solution to these equations is given by a linear dilaton vacuum 30]:

$$
\varphi=8 \widetilde{m}_{\psi} x^{1}
$$

8 Strictly speaking, $\Delta$ and $f$ can not be constant in a generic compactification on a Calabi-Yau space $X$. Indeed, the warp factor has to satisfy Laplace equation in eight compact dimensions. However, integrating over a compact manifold $X$, the terms containing derivatives of these functions with respect to internal coordinates average to zero. 
where the constant spinor $\epsilon$ is supposed to satisfy:

$$
\left(\gamma^{1}+1\right) \epsilon=0
$$

This dilaton background spontaneously breaks Poincare invariance in two dimensions (down to time translations) and half the supersymmetries preserved by Calabi-Yau fourfold. The physics of these vacua is very interesting and quite subtle [30]. Here we shall not discuss it any further, and return to the test of supersymmetry conditions for the new solutions found in section 5 .

As in the solutions (5.5), we assume that gravitino fields are massless. This implies that dilaton depends only on the internal coordinates $x^{m}$, but not on the two-dimensional coordinates $x^{\mu}, c f$. ([.20). This dependence can be determined from eq. ([.15) and from the variation of the dilatino (I.12) that gives:

$$
\left(\partial_{m} \varphi\right)-\frac{\Delta}{6} e^{-\frac{1}{2} \varphi}\left(\partial_{m} f\right)=0
$$

The solution looks like:

$$
e^{\varphi}=\Delta^{-9}, \quad f=\frac{108}{11} \Delta^{-11 / 2}
$$

As we discussed above, the other components of ([.12) are equivalent to the linear combination of ([.16) and ([.19), so that we may focus only on the latter two conditions and no longer worry about the variation of the dilatino - it will vanish automatically, as long as the scalar functions $f\left(x^{m}\right), \Delta\left(x^{m}\right)$, and $\varphi\left(x^{m}\right)$ obey (I.23). In what follows we assume that this is always the case, and suppress the dependence on these functions in order to make a comparison to the result of the previous subsection, where we implicitly set the string coupling constant, $g_{\mathrm{st}}=e^{\varphi}$, equal to unity.

The remaining components of the supersymmetry conditions (1.16) and (‥19) depend on the particular choice of Ramond-Ramond fluxes. We shall analyze these conditions for the background fluxes given by the wedge products of the Kähler form $\mathcal{K}$ as in (5.1). Then, the only non-trivial components of ([.16) and ([.19) correspond to singlet representation of $S U(4)$ and have a very simple form:

$$
\left(-4 M+28 F_{\mu \nu} \gamma^{\mu \nu} \Gamma_{11}+G\right) \eta=0
$$

and

$$
\left(12 M+12 F_{\mu \nu} \gamma^{\mu \nu} \Gamma_{11}+q k\right) \eta=0
$$


Notice, that with our choice of the two-form flux $F$ allows to rewrite these supersymmetry conditions without $\Gamma_{11}$, which is related to the fact that $\check{F}^{(8)}$ has a real contribution to the effective superpotential (2.6). In fact, using the standard gamma-matrix algebra and the projection relation ([1.6) we get the following relation:

$$
F_{\mu \nu} \gamma^{\mu \nu} \Gamma_{11} \eta=n_{8} \eta
$$

where we identified $F_{\mu \nu}$ components of the 2-form field strength with the Hodge dual 8form flux $\check{F}^{(8)}$ in (5.1). Using the relation (I.26) and the following properties of $S U(4)$ holonomy manifolds [2]:

$$
\gamma_{\bar{a} \bar{b} c d} \zeta=3 \mathcal{K}_{[\bar{a} c} \mathcal{K}_{\bar{b} d]} \zeta, \quad *(\mathcal{K} \wedge \mathcal{K})=\mathcal{K} \wedge \mathcal{K}
$$

one can put (1.24) - (1.25) in the form of two algebraic equations:

$$
-4 n_{0}+28 n_{8}+72 n_{4}=0 \text { and } 12 n_{0}+12 n_{8}+72 n_{4}=0
$$

Now it is easy to solve these equations:

$$
n_{0}=n_{8}=-3 n_{4}
$$

This solution is equivalent to (5.5) that we found earlier analyzing superpotential terms in the effective two-dimensional theory. Therefore, microscopic analysis of the supersymmetry conditions in massive Type IIA supergravity gives us an independent evidence for the expressions (2.4) and (2.6).

Finally, we explain that another set of supersymmetry transformations ([.11) and (I.12) with a spinor $\eta$ of negative chirality leads to complex conjugated supersymmetry conditions. Notice that according to ([.6) the ten-dimensional chirality of the spinor $\eta$ is directly related to the two-dimensional chirality of the spinor $\epsilon$. If in our calculation we used the spinor $\epsilon$ of negative chirality, we would obtain the complex conjugated supersymmetry conditions. To see this we note that $\Gamma_{11}$ appears in (I.11) and (I.12) only in the terms that contain $F_{M N}$ or $H_{M N P}$. However, the three-form $H$ always comes contracted with two-dimensional gamma-matrices, so that we have extra $\gamma_{3}$ factor due to the specific form of the ansatz ([.5). Therefore, the terms with $H$ do not change the sign if we change the chirality of $\eta(=$ chirality of $\epsilon)$. This is not the case for the terms proportional to the 
internal components of the two-form $F$ and for the terms linear in $G_{\mu \nu m n}$ which have the same gamma-matrix structure:

$$
G_{\mu \nu m n} \Gamma^{\mu \nu m n} \eta=-G_{01 m n} \gamma^{m n} \Gamma_{11} \eta
$$

We conclude that the supersymmetry conditions for the spinors of negative chirality differ only by signs of all the terms with $F_{m n}$ and $G_{\mu \nu m n}$. These conditions should be compared with the variation of complex conjugate superpotential (2.6). In our examples of the form (5.1), these conditions are automatically satisfied because the fields $F_{m n}$ and $G_{\mu \nu m n}$ were assumed to vanish in the background.

\section{Acknowledgments}

I would like to express my gratitude to S. James Gates and especially to Edward Witten for helpful discussions and constant encouragement. The work was supported in part by the Caltech Discovery Fund, grant RFBR No 98-02-16575 and Russian President's grant No 96-15-96939. 


\section{References}

[1] R. Harvey and H.B. Lawson, Jr., "Calibrated geometries", Acta Math. 148 (1982) 47.

[2] K. Becker, M. Becker, D.R. Morrison, H. Ooguri, Y. Oz, Z. Yin, "Supersymmetric Cycles in Exceptional Holonomy Manifolds and Calabi-Yau 4-Folds", Nucl.Phys. B480 (1996) 225.

[3] G.W. Gibbons and G. Papadopoulos, "Calibrations and Intersecting Branes", Commun. Math. Phys. 202 (1999) 593.

[4] J.P. Gauntlett, N.D. Lambert, P.C. West, "Branes and Calibrated Geometries", Commun.Math.Phys. 202 (1999) 571.

[5] B. S. Acharya, J. M. Figueroa-O'Farrill, B. Spence, "Branes at angles and calibrated geometry", JHEP 9804 (1998) 012.

[6] J.M. Figueroa-O'Farrill, "Intersecting brane geometries", hep-th/9806040.

[7] G. Papadopoulos and A. Teschendorff, "Grassmannians, Calibrations and Five-Brane Intersections", hep-th/9811034.

[8] J. Gutowski, G. Papadopoulos, P. K. Townsend, "Supersymmetry and Generalized Calibrations", hep-th/9905156.

[9] R. Minasian, D. Tsimpis, "M5-branes, Special Lagrangian Submanifolds and sigmamodels", hep-th/9906190.

[10] R. C. McLean, "Deformations of Calibrated Submanifolds", Comm. Anal. Geom. 6 (1998) 705.

[11] N. Hitchin, "The Moduli Space of Special Lagrangian Submanifolds", Ann. Scuola Norm. Sup. Pisa Cl. Sci. (4) 25 (1997), 503.

[12] A. Strominger, S.-T. Yau, E. Zaslow, "Mirror Symmetry is T-Duality", Nucl.Phys. B479 (1996) 243.

[13] C. Vafa, "Extending Mirror Conjecture to Calabi-Yau with Bundles", hep-th/9804131.

[14] D. Joyce, "On counting special Lagrangian homology 3-spheres", hep-th/9907013.

[15] R. Gopakumar, C. Vafa, "M-Theory and Topological Strings - I, II", hep-th/9809187, hep-th/9812127.

[16] B. S. Acharya, "M theory, Joyce Orbifolds and Super Yang-Mills", hep-th/9812205.

[17] J.A. Harvey and G. Moore, "Superpotentials and Membrane Instantons", hepth/9907026.

[18] K. Dasgupta, G. Rajesh, S. Sethi, "M Theory, Orientifolds and G-Flux", hepth/9908088.

[19] S. Gukov, C. Vafa and E. Witten, "CFT's From Calabi-Yau Four-folds", hepth/9906070.

[20] S.J. Gates, Jr., S. Gukov and E. Witten, "Two Two-Dimensional Supergravity Theories from Calabi-Yau Four-Folds", Nucl.Phys. B584 (2000) 109. 
[21] S. Sethi, C. Vafa, and E. Witten, "Constraints on Low-Dimensional String Compactifications", Nucl. Phys. B480 (1996) 213.

[22] E. Witten, "On Flux Quantization In M-Theory And The Effective Action", J. Geom. Phys. 22 (1997) 1.

[23] J. Polchinski and A. Strominger, "New Vacua for Type II String Theory," Phys. Lett. B388 (1996) 736.

[24] L. J. Romans, "Massive N=2A Supergravity in Ten Dimensions", Phys.Lett. 169B (1986) 374.

[25] W. Lerche, "Fayet-Iliopoulos Potentials from Four-Folds", JHEP 9711 (1997) 004.

[26] H. Ooguri, Y. Oz, Z. Yin, "D-Branes on Calabi-Yau Spaces and Their Mirrors", Nucl.Phys. B477 (1996) 407.

[27] K. Becker, M. Becker, "M-Theory on Eight-Manifolds", Nucl.Phys. B477 (1996) 155.

[28] E. Witten and J. Bagger, "Quantization Of Newton's Constant In Certain Supergravity Theories", Phys. Lett. 115B (1982) 202.

[29] S.J. Gates, Jr., M.T. Grisaru and M.E. Wehlau, "A Study of General 2D, N=2 Matter Coupled to Supergravity in Superspace", Nucl.Phys. B460 (1996) 579.

[30] N. Berkovits, S. Gukov, and B. Vallilo, to appear.

[31] A. Strominger, "Special geometry", Comm. Math. Phys. 133 (1990) 163.

[32] G. Tian, "Smoothness of the universal deformation space of compact Calabi-Yau manifolds and its Petersson-Weil metric", in Mathematical aspects of string theory, World Sci. Publishing, Singapore, 1987, p. 629.

[33] E. Witten, "Non-Perturbative Superpotentials In String Theory", Nucl.Phys. B474 (1996) 343.

[34] E. Witten, "Constraints on Supersymmetry Breaking", Nucl.Phys. B202 (1982) 253.

[35] M. Green, J.H. Harvey and G. Moore, "I-Brane Inflow and Anomalous Couplings on D-branes", Class. Quant. Grav. 14 (1997) 47.

[36] M. Gromov, "Pseudoholomorphic curves in symplectic manifolds", Inv.Math. 82 (1985) 307.

[37] E. Witten, "Two-dimensional gravity and intersection theory on moduli space", Surveys in Diff. Geom. 1 (1991) 243.

[38] A. Strominger, C. Vafa, "Microscopic Origin of the Bekenstein-Hawking Entropy", Phys.Lett. B379 (1996) 99.

[39] J. Maldacena, A. Strominger, E. Witten, "Black Hole Entropy in M-Theory", JHEP 9712 (1997) 002.

[40] C. Vafa, "Black Holes and Calabi-Yau Threefolds", Adv.Theor.Math.Phys. 2 (1998) 207.

[41] M. Banados, M. Henneaux, C. Teitelboim, J. Zanelli, "The Black Hole in Three Dimensional Space Time", Phys.Rev.Lett. 69 (1992) 1849.

[42] S. Carlip, "The (2+1)-Dimensional Black Hole", Class.Quant.Grav. 12 (1995) 2853. 
[43] M. Kontsevich, "Homological Algebra of Mirror Symmetry", alg-geom/9411018.

[44] M. Gross, "Special Lagrangian Fibrations I: Topology", alg-geom/9710006.

[45] E. Bergshoeff, M. de Roo, M. B. Green, G. Papadopoulos, P. K. Townsend, "Duality of Type II 7-branes and 8-branes", Nucl.Phys. B470 (1996) 113. 